\title{
Civilisations
}

Revue internationale d'anthropologie et de sciences

humaines

49 | 2002

Pain, fours et foyers des temps passés

\section{Les céréales cultivées par les populations Néo et Enéolithiques de la Moldavie}

\section{Felicia Monah et Dan Monah}

\section{(2) OpenEdition \\ Journals}

Édition électronique

URL : http://journals.openedition.org/civilisations/1366

DOI : 10.4000/civilisations. 1366

ISSN : 2032-0442

\section{Éditeur}

Institut de sociologie de l'Université Libre de Bruxelles

\section{Édition imprimée}

Date de publication : 3 juin 2002

Pagination : 67-76

ISBN : 0009-8140

ISSN : 0009-8140

Référence électronique

Felicia Monah et Dan Monah, «Les céréales cultivées par les populations Néo et Enéolithiques de la Moldavie », Civilisations [En ligne], 49 | 2002, mis en ligne le 01 juin 2005, consulté le 01 mai 2019.

URL : http://journals.openedition.org/civilisations/1366; DOI : 10.4000/civilisations. 1366

Ce document a été généré automatiquement le 1 mai 2019.

(c) Tous droits réservés 


\title{
Les céréales cultivées par les populations Néo et Enéolithiques de la Moldavie
}

\author{
Felicia Monah et Dan Monah
}

Les auteurs sont heureux d'exprimer toute leur reconnaissance à Dr. Ph. Marinval qui a bien voulu assumer la révision du texte français.

\section{Cadre géographique}

La province historique de la Moldavie comprend le territoire borné à l'Ouest par les C arpates orientales, au Sud par le Bas-Danube et le littoral de la mer Noire. A l'Est et au N ord, la frontière est marquée par les cours du Nistru et celui du Ceremus (fig. 1). A cause de l'histoire, le territoire de la Moldavie est aujourd'hui divisé par le Prut en deux régions politiques. La part occidentale, qui se situe entre les Carpates et le Prut est connue sous le nom de Moldavie et la zone orientale, entre le Prut et le Nistru représente la République de la Moldavie. La dénomination historique consacrée de cette zone, qui sera celle que nous allons employer au cours de ce texte, est la Bessarabie. 


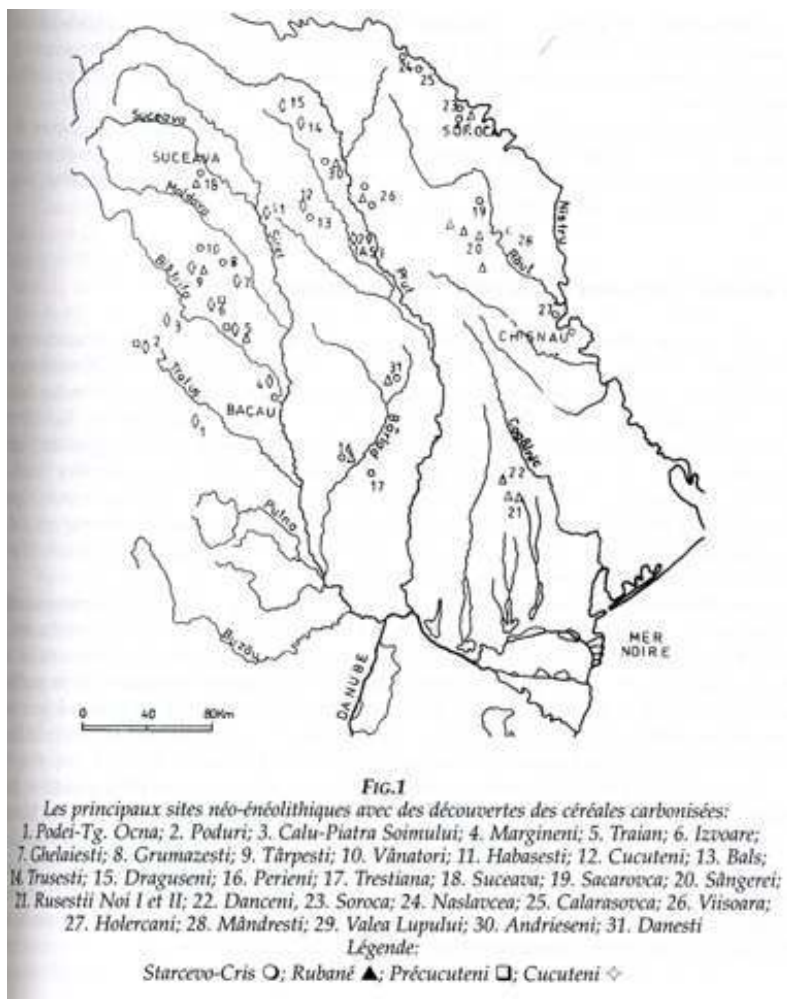

2 Le relief de la province est varié. Il comprend la chaîne des Carpates orientales, région subcarpatique caractérisée par des hautes collines et un grand plateau, dénommé Plateau moldave. Cette dernière formation est très développée. Elle s'étend au-delà du Nistru, jusqu'au Bug. Les plaines ne sont pas assez étendues et elles se situent surtout au Sud de la province. Les systèmes hydrographiques du Siret, du Prut et du Nistru, cours d'eau qui proviennent des Carpates, arrosent à peu près toute la superficie du Plateau moldave. La région montagneuse et subcarpatique possède un réseau dense de cours d'eau qui diminue vers le sud de la province. Le climat de la Moldavie est tempéré-continental soumis à de profondes influences estiques qui sont toutefois atténuées par les Carpates, l' Atlantique et la Méditerranée. La Moldavie se situe dans une zone où les précipitations sont relativement réduites et à compensation pluvio-métrique irrégulière. Cependant, les régions montagneuses et subcarpatiques présentent une humidité excédentaire. Les $\mathrm{S}$ ubcarpates et le plateau moldave se caractérisent par des sols argilo-fluviaux bruns podzoliques et des sols podzoliques. Dans la zone de collines se rencontrent, parsemés, des sols argilo-fluviaux gris et les tchernozioms lévigés, dont la fertilité est supérieure.

Les tchernozioms, d'une grande fertilité, prédominent dans les plaines. En général, les conditions climatiques et la fertilité du sol offrent à la Moldavie un potentiel agricole élevé (Mihailescu 1969 : 117, Sircu 1971 : 14 ss, Topor 1963, Berg 1918, : 4-11, Odud 1955 7-13 et Chitu 1975).

\section{Historique des recherches archéobotaniques}

Les recherches archéobotaniques disposent d'une certaine tradition en Moldavie. Dans les années 30, Radu Popovici, disciple du Professeur Netolitzky, a débuté toute une série de déterminations anthracologiques. Soutenue comme thèse de doctorat à l'Université de $\mathrm{C}$ ernauti (Popovici 1932: 229-250, $1933: 244-249$ et 1935), cette recherche a concerné les 
forets paléolithiques et néolithiques du nord de la Roumanie. C'est en 1946 que Traian S avulescu a conduit la première investigation approfondie concernant les macrorestes végétaux d'un habitat énéolithique (Savulescu 1946:37-42). Il existe toute une série d'autres déterminations, qui malheureusement ont été effectuées par des agronomes qui n'étaient pas initiés à l'archéobotanique (Dumitrescu et al. 1954 : 506).

Au début des années 60 , Zoia Janusevic de Chisinau a entamé un programme de recherche pour déterminer des macrotestes végétaux de la Bessarabie. La botaniste a réaliser des contributions importantes et reconnues dans le domaine de la morphologie et des clefs de détermination pour les plantes cultivées de la préhistoire. Comme fort peu de dépôts de céréales carbonisées furent mis au jour en Bessarabie, notre collègue de Chisinau s'est consacrée à l'étude des empreintes des semences imprimées dans la céramique et le torchis (Janusevic 1976 : 8-10).

6 Dans les années 80, une recherche similaire concernant la détermination des macrorestes végétaux néo-énéolithiques, a été initiée en Roumanie par Marin Cîrciumaru (Wasylikova et al. 1991 : 208). Dans l'ouest de la Moldavie, les archéobotanistes ont eu la chance de travailler sur de nombreux dépôts très riches en céréales carbonisées. A présent, nous estimons possible de proposer certaines appréciations concernant les principales espèces de céréales cultivées et leurs emplois par les populations néo-énéolithiques de la $\mathrm{M}$ oldavie.

\section{Les plantes cultivées dans les cultures de $S$ tarcevo- Cris et Rubané}

7 L'existence d'un Néolithique acéramique à Soroca n'est pas prouvée. Actuellement, il n'en n'est même plus question. En l'état actuel des recherches archéobotaniques moldaves, les premiers habitants néolithiques de l'espace carpato- nistrien appartiennent au grand complexe culturel de Starcevo-Cris. Certains archéologues bessarabiens soutiennent l'existence, à l'Est de la province, le long du Nistru, d'une culture autochtone nommée B ug-Nistru (Markevich 1974 et Larina 1994 : 42-46), qui, selon nous, représente seulement un aspect acculturé de la culture de Starcevo- Cris. Cette dernière, selon les datations 14c, s'échelonne entre 6100 et 5500 B.C. (Gimbutas 1991, tabl. 6), tandis que la culture de Bug N istru est datée de 6400-5500 B.C. (Gimbutas 1991, tabl. 6 et Larina $1994: 43$ ).

Dans les habitats de la culture Starcevo-Cris et sa variante Bug-Nistru on a déterminé la présence du blé amidonnier (Triticum dicoccum) et de l'épeautre (T. Spelta) ainsi que celle de l'orge (Hordeum vulgare et $H$. Vulgare var. coeleste) (Janusevic $1986: 4-8$ et $1975: 33$ ). Il est important de signaler aussi la détermination de deux empreintes d'avoine (Avena sp.). Z. Janusevic estime que Triticum dicoccum constituait la culture prépondérante de la civilisation de Starcevo- Cris en Bessarabie (Janusevic $1986: 11$ et $1983: 267$ ).

Dans le dernier quart du vie millénaire B.C., pénètrent sur le territoire de la Moldavie les auteurs de la céramique rubanée qui, à notre avis, vont cohabiter pour quelques temps avec les tribus Starcevo- Cris. Les communautés de la céramique rubanée, datée entre 5300-5070 B.C. (Gimbutas 1991 tabl. 4), semble être enclavées dans le territoire StarcevoCris. Mais les nouveaux habitants de la Moldavie semblent avoir un rôle culturel particulier, parce que du mélange des éléments rubanés avec ceux de Boian-Giulesti, parvenus du Sud, de la Valachie, va naître la culture Precucuteni (Marinescu-Bilcu 1974: 110). Dans peu d'habitats rubanés fouillés, on a mis en évidence la culture de Triticum 
dicoccum, T. spelta et on a signalé la présence du millet (Panicum miliaceum) et de l'avoine ( Avena sativa) (Cîrciumaru 1991 : 230).

\section{Les plantes cultivées dans la culture de Precucuteni}

Pour la culture de Precucuteni qui s'étend sur presque tout le territoire de la Moldavie (4780-4340 B.C.), on a obtenu de nombreuses et intéressantes données sur les céréales cultivées à cette époque là.

11 Dans les niveaux attribués à la culture Precucuteni du Tell de Poduri, on a découvert 17 importants dépôts de céréales carbonisées. On a récupéré $34 \mathrm{~kg}$ de caryopses carbonisés, seulement au sein des silos de la construction n 44 (Cârciumaru-Monah $1985:$ 700-706 et Cârciumaru-Monah 1987 : 169-171). Pourtant cette découverte n'est pas si importante en comparaison avec celle de Teiu, datant de culture de Gumelnita. Plus de $2000 \mathrm{~kg}$ de céréales carbonisées furent récupérés des silos de ce gisement (Cârciumaru 1990 : 65).

On enregistre certaines préférences régionales envers certaines espèces de blé et d'orge pendant le Precucuteni. Ainsi, dans la région Ouest de la Moldavie on cultivait surtout $T$ riticum aestivum, T. dicoccum et T. monococcum tandis que T. spelta et T.compactum semblaient avoir une aire plus réduite, voire ne pas avoir été cultivées délibérement et ne devaient constituer que des mauvaises herbes des champs des autres céréales. Au cours de la même période, entre les Carpates et le Prut, on cultivait surtout Hordeum vulgare et $H$. vulgare nudum (Cârciumaru-Monah 1987 : 169-172).

En Bessarabie, la première place était occupée par Triticum monococcum suivi de T. spelta et de T. dicoccum. Pour cette région on signale aussi T. compactum. et Hordeum vulgare. Cette dernière espèce est mentionnée dans trois habitats, mais c'est $H$. vulgare var. coeleste qui prédomine. Quoique Panicum miliaceum soit signalé dans les trois sites, il ne semble pas occuper une place importante parmi les céréales cultivées par la population $\mathrm{P}$ recucuteniènne (Janusevic 1976 tabl. 1 et passim).

14 La culture Precucuteni qui précède la culture Cucuteni date par $14 \mathrm{C}$ entre 4660 et 3700 B.C .(Monah $1987: 75-77$ et fig. 1, Gimbutas $1991:$ 458).

\section{Les plantes cultivées dans la culture de Cucuteni}

Dans la première phase Cucuteni A on préfère, semble-t-il exploiter T. dicoccum et $T$. aestivum. T. monococcum et T. spelta, bien que mentionnés, semblent avoir une moindre importance (Cârciumaru-Monah 1987 : 168-173, Wasylikova et al. 1991 : 210-213). On y signale aussi la présence de T. aestivo-compactum, mais dans des pourcentages réduits. Extrêmement intéressante du point de vue botanique est la présence des $T$. dicoccoides et de T.durum (le blé dur) (Cârciumaru-Monah 1987: 168-173, Wasylikova et al. 1991: 210-213). Si la première espèce peut être interprétée comme une dégénérescence de certains exemplaires de $T$. dicoccum, la seconde suggère que le blé dur n'était pas encore semé de façon monospécifique. En Bessarabie, on enregistre les mêmes prépondérances de $T$. dicoccum suivi de T. monococcum et de T. spelta. Le T. aestivo-compactum est également attesté, sans toutefois être très répandu (Cârciumaru-Monah 1987 : 168-173, Wasylikova et al. 1991 : 210-213). L'importance de l'orge devait être similaire à celle du blé. On en a découvert plusieurs dépôts importants dans les habitats de la phase Cucuteni A. En M oldavie occidentale, on cultivait surtout Hordeum vulgare et $H$. vulgare var. nudum (l'orge 
polystique à grains nus), mais on mentionne aussi Hordeum distichon (l'orge à deux rangs). Dans la même région on signale également la présence d'Avena sativa (l'avoine cultivée) et deSecale cereale (le seigle). Qui n'étaient pas, semble-t-il, semés en cultures propres. Panicum miliaceum (le millet commun) est également attesté, mais dans une fréquence et une importance économique réduite (Cîrciumaru-Monah 1987 : 168-173 et Wasylikova et al. 1991 : 210-213). Pourtant, provenant des fouilles conduites avant la seconde guerre mondiale à Calu, fut mis au jour un fragment d'une galette. Celle-ci est constituée d'une farine de millet, puisqu'on a observé des grains de millet lors de sa découverte (Vulpe 1941: 21, Matasa 1940: 16 et 28). Malheureusement, cette pièce exceptionnelle a été perdue pendant la guerre.

En Bessarabie, on signale dans la phase Cucuteni A, outre la culture de Hordeum vulgare et H. vulgare var. coeleste, la présence de l'avoine (Avena sativa) et un emploi plus important du millet (Panicum miliaceum) (Janusevic 1976 : tabl. 1 et passim).

Enfin, dans la dernière phase (B) de la culture Cucuteni dans la partie Ouest de la province, on préfère la culture de Triticum compactum et T. aestivum, accompagnés dans une moindre mesure par T. dicoccum et T. monococcum. Moins répandus encore sont, semble-t-il, les espèces T.spelta et T.durum (Cîrciumaru-Monah 1987: 173). Ces modifications pourraient être dues à certains changements climatiques; car les cultures du seigle et du millet semblent être alors de plus en plus répandues.

Tableau 1 - La fréquence des espèces des céréales dans les cultures néo-énéolithiques de la Moldavie. Légende: +++ =abondant, ++ = fréquent $+=$ occasionnel, - = rare

\begin{tabular}{|c|c|c|c|c|c|c|}
\hline \multirow{2}{*}{\multicolumn{2}{|c|}{\begin{tabular}{|l|} 
Piriodes \\
Cultures/ \\
Ptetions
\end{tabular}}} & \multicolumn{2}{|l|}{ Néb, ancires } & \multirow{2}{*}{\begin{tabular}{|l|} 
Frecucuteni \\
Oovest Moldavie \\
\end{tabular}} & \multirow[b]{2}{*}{$\begin{array}{c}\text { entre Carpotes } \\
\text { et Prot }\end{array}$} & \multirow[b]{2}{*}{ Bessaratic } \\
\hline & & Stacceto-Cris & Rubanè & & & \\
\hline \multicolumn{2}{|l|}{ Datations (en B,C.) } & $6100-5500$ & $5300-5070$ & & $4780-4340$ & \\
\hline Amidonnier & Trikicum ficestram & $\omega$ & H & + & & t+ \\
\hline Epenuter & Iridicum spelia & H & H & & & + \\
\hline Orinevitue & Honitrum videare & +4 & & & H & + \\
\hline Orge mix & Handeum widgare nar. coclaste & H & & & & \\
\hline Avoine & fwenasp & + & + & & & \\
\hline Millet commun & Panicum mulhacreum & & + & & & + \\
\hline Froment & Triniticum exstivem & & & H & & \\
\hline Evgrain & Trificum minnocacciam & & & $\rightarrow+$ & & H+H \\
\hline Blé hérisson & Triticicues compactom & & & + & & + \\
\hline Orge nue & Ulorikum vulgare ser. muchum & & & & $+1+$ & \\
\hline Froment TBlé hárissuen: & Trinificum sesfivo-cianpaithum & & & & & \\
\hline Seigle & Secole cerenale & & & & & \\
\hline Amidoonict sauvage & Triviticuit dicrocooides & & & & & \\
\hline Ble dur & Trititicum thuram & & & & & \\
\hline Awoine cultives & Arene sativur & & & & & \\
\hline Orge ditatique & Hondremer davichan & & & & & \\
\hline
\end{tabular}

En Bessarabie, les semailles de T. monococcum, $T$. dicoccum et $T$. spelta sont prépondérantes, mais on y signale aussi T. compactum (Janusevic 1976: 20-103). Même en Bessarabie, en dehors du blé, l'orge semble avoir assuré le besoin en céréales des Cucuténiens (Janusevic 1976 : 103-127). Dans cette région, Panicum miliaceum semble avoir été largement employé (Janusevic 1976 : 153-156).

\section{Données agro-écologiques de quelques plantes cultivées}

Pour conclure, on peut apporter quelques précisions sur les céréales cultivées par les populations néo-énéolithiques de la Moldavie. En ce qui concerne le genre Triticum, les espèces les plus employées, à l'Ouest de la province, sont les espèces nues comme 
T. aestivum (le froment) et T. compactum (le blé hérisson). Le froment a une productivité élevée et est facile à égrener. Il fournit une farine de bonne qualité (Janusevic 1986 : 48). T. compactum est résistant à la sécheresse. Il est précoce et sans prétentions. Sa productivité est plus faible que celle de T. aestivum, mais ses grains se conservent plus longtemps. Des deux, le froment fournit la plus grande quantité de farine (Janusevic 1976 : 79). Triticum spelta est une variété de blé tendre à grains vêtus. Il est moins exigeant sur les conditions climatiques et la valeur des sols, que les autres espèces. On l'emploie encore en Fergana ${ }^{1}$ dans la fabrication des tourtes considérées comme ayant de hautes qualités nutritives. N.I. Vavilov précise que dans cette région les épis d'épeautre étaient moissonnés à l'aide des ciseaux (Janusevic 1976: 73). L'amidonnier (Triticum dicoccum) encore appelé épeautre de Tartarie a une grande adaptabilité aux sols très humides ou secs. Pendant la grande sécheresse de 1921 sur la Volga, T. dicoccum a été la seule céréale qui ait résistée (Janusevic 1976 : 56-57). Les grains d'amidonnier sont riches en protéines ( Janusevic 1976 : 56-57). Dans les habitats cucuténiens on le gardait sous forme d'épis, ce qui demande des dépôts de grande taille. Dans les livres de cuisine russes du XVIIIe siècle, la bouillie de $T$. dicoccum était recommandée comme étant "extrêmement facile à digérer " et les pâtissiers l'apprécient pour sa farine blanche. Pour le pain, il est recommandé d'employer un mélange avec de la farine de seigle (Janusevic 1976 : 57-59).

En ce qui concerne l'orge, il faut remarquer que Hordeum vulgare var. nudum a un contenu élevé d'aminoacides et de protéines, ces facteurs donnent au pain des propriétés particulières (Janusevic 1976 : 116-117). Les espèces vêtues d'orge sont plus difficiles à employer dans l'alimentation des humains (Janusevic 1976 : 117).

21 Bien que les dépôts des habitats Precucuteni-Cucuteni contiennent d'habitude des mélanges de blé et d'orge, toute une série d'informations archéologiques et botaniques montre qu'on pratique une sélection artificielle des céréales, d'après l'espèce ou le genre, pendant l'Enéolithique de la Moldavie (Monah et Monah 1987 : 158).

Dans quelques habitats de la culture Cucuteni on a découvert des glands. Probablement ceux-ci étaient moulus et la farine ainsi obtenue était mélangée avec celle de blé ou d'orge. Pendant la grande famine qu'a connue la Moldavie au cours des années 1946-47, causées par la sécheresse et la confiscation par l'armée soviétique d'occupation de toute la récolte de céréales, dans les villages moldaves on confectionnait un mélange de farine de glands et d'infime quantité de farine d'orge ou de blé. Bien que les glands aient été mis en eau plusieurs jours pour éliminer le tanin, selon une ancienne recette, le pain et les galettes demeuraient amers et peu nutritifs.

Il nous semble particulièrement important de signaler que dans la religion des tribus $C$ ucuteni- Tripolye, les céréales, surtout le blé et l'orge, occupaient un rôle important. Sur les vases et les statuettes figurent des épis incisés ou peints. Dans la pâte de certaines statuettes anthropomorphes on a introduit des caryopses d'orge et de blé et même quelques exemplaires avaient dans leur pâte de la farine d'orge (Bibikov: 204-213 et fig. 69-72). Cette pratique, connue aussi chez les tribus rubanées (Höckmann : 89-98), laisse supposer qu'il y a eu un début de cristallisation d'un dogme de type de la transsubstantiation qui pouvait remplacer les sacrifices humains (Monah 1995 : 21). 


\section{BIBLIOGRAPHIE}

BERG S.L., 1918 Bessarabija. Petrograd.

BIBIKOV S.N., 1953. Poselenie Luka-Vrubleveckaja na Dnestre.In : Materialy i issledovanija po

arkheologii SSSR, 38, Moscou-Leningrad

CHITU C., 1975, Relieful si solurile României. Craiova.

CÂRCIUMARU M., 1990. Etude paléobotanique pour les habitats néo-lithiques et énéolithiques de $\mathrm{R}$ oumanie. Acta Interdisciplinaria Archaeologica, 7 : 61-73.

CÂRCIUMARU M. et MONAH F., 1985. Raport preliminar privind semin-tele carbonizate de la PoduriDealul Ghindaru, jud Bacau, In : Memoria Antiquitatis, IX-XI : 699-708

CÂRCIUMARU M. et MONAH F., 1987 Déterminations paléobotaniques pour les cultures Precucuteni et Cucuteni, Iasi : 167-174. In : La civilisation de Cucuteni en contexte européen.

DUMITRESCU V.L. et al., 1954. Habasesti. Monografie archeologica, Bucuresti

GIMBUTAS M., 1991. The civilization of the Goddess. The World of the Old Europe. San Francisco.

HockMANN O., 1987. Gemeinsamkeiten in der Plastik der Linearkeramik und der Cucuteni-Kultur, Iasi : 89-98. In : La civilisation de Cucuteni en contexte européen

JANUSEVIC Z.V., 1975. Fossil remains of cultivated plants in the south west of the soviet union. Folia quaternaria, 46, Kracow : 23-20

JANUSEVIC Z.V., 1976. Kuloturnye rastenija Jugo-Zarada SSSR po poleobotaniceskimissledovanijam, K isinev

JANUSEVIC Z.V., 1984. The specific composition of wheat finds from ancient agricultural centres in the USSR. In : W. van Zeist et W.A. Casparie (éd.). Plants and Ancient Man. Studies in P alaeoethnobotany. Procceding of $6^{\circ}$ Symposium IWGP. Balkema, Rotterdam : 267-276.

JANUSEVIC Z.V., 1986 Kuloturnye rastenija Severnogo Pricernomoroja., Kisinev.

LARINA O., (1994). Neoliticul pe teritoriul Republicii Moldova. In : Thraco-Dacica, 11 (1-2) : 41-66.

MARINESCU-BILCU S., 1974 Cultura Precucuteni pe teritoriul României. Bucuresti.

MARKEVIC V., 1974 Bugo-Dnestrovskaja kulotura na teritorii Moldavii. Kisinev.

MATASA C., (1940). Cercetari din preistoria judetului Neamt. In : Buletinul Comisiunii Monumentelor I storice, 97, Valenii de Munte :1-41.

MIHAILESCU V., (1969). Geografia fizica a României. Bucuresti.

MONAH D., 1987 La datation par ${ }^{14} \mathrm{C}$ du complexe Ccucuteni-Tripolie.In : La civilisation de Cucuteni en contexte européen. Iasi : 67-80.

MONAH D., 1995 Plastica antropomorfa a culturii Cucuteni. Iasi.

MONAH D. et MONAH F., 1987 Plante folosite de populatiile neo-eneolitice de pe teritoriul Moldovei. In : Culegere de Studii si Articole de Biologie, 3, Iasi :156-159.

ODUD L.A., 1955 Moldavskaja SSSR, Moscou. 
POPOVICI R., 1932 Beiträge zur Walgeschichte Nord Rumänien. In : Buletinul Facultatii de Stiinte din C ernauti (BFSC), VI : 229-250.

POPOVICI R., 1933 Ein Weitterer Beitrag zur Walgeschichte unseres Landes. In : Buletinul Buletinul F acultatii de Stiinte din Cernauti (BFSC), VII : 244-249.

PopovicI R., 1935 Padurile Paleo- si Neolitice din Nordul Romaniei. Cernauti.

SAVULESCU T., 1946 La flore.In : Matasa C. Frumusica. Village préhistorique à céramique peinte dans la M oldavie du Nord, Roumanie. Bucuresti : 37-42.

SIRCU I., 1971 Geografia fizica a Republicii Socialiste România. Bucuresti

TOPOR N., 1963 Ani ploiosi si secetosi in Republica Populara România. Bucuresti

VULPE R., (1941). Les fouilles de Calu. In : Dacia, 7-8 : 13-68.

Wasylikowa K., CÂRciumaru M., Hajnalov E., Hartynyi P., PaskeVic G.A. et JanuseVic ZV.., 1991 E ast-Central Europe.In : W. Van Zeist, Wasylikowa K et Bhere K.-E. (dir.). Progress in Old World $P$ alaeoethnobotany. Balkema, Rotterdam : 207-239.

\section{NOTES}

1. Fergana: vallée qui s'étend sur l'Ousbékistan, le Kirghizistan et le Tadjikistan.

\section{RÉSUMÉS}

L'article présente une image de l'introduction et de la culture des céréales durant le Néolithique et l'Enéolithique de la Moldavie et de la Bessarabie (République de Moldavie), provinces roumaines situées à l'Est des Carpates orientales. Un bref historique des recherches archéobotaniques consacrées à ces régions est retracé. Les auteurs remarquent que des préférences pour certaines espèces de blé et d'orge, sont manifestent, selon l'époque étudiée (Néolithique ou Enéolithique), mais aussi la géographie (Est ou Ouest de la province). Pour finir, quelques considérations sur les qualités agroécologiques de diverses céréales sont proposées dans le cadre du Néolithique et l'Enéolithique de Moldavie.

This article describes the introduction and cultivation of cereal crops during the Neolithic and Eneolithic periods, East of the Carpathian Moutains, in the province of Moldavia (Romania) and the Bessarabia (Rep. of Moldovia). A succint history of archaeobotanical research for the prehistoric period of this region is provided. The autors note a difference in the temporal and geographic preferences for certain species of wheat and barley for the neolithic and eneolithic periods and in Moldavia and the Bessarabia. The authors draw conclusions on the nutritional quality of differer species of cultivated cereals during the Neo-Eneolithic period in the Earstern of the Carpathians. 
INDEX

Mots-clés : Pain, céréales, Moldavie, néolithique, énéolithique, archéobotanique

Keywords : Bread, cereals, Moldavia, Neolithic, Eneolithic, archaeobotany

\section{AUTEURS}

FELICIA MONAH

Département d'Archéobotanique, Institutul de Arheologie, Iasi, România.

\section{DAN MONAH}

Département de Préhistoire, Institutul de Arheologie, Iasi, România. 